\title{
Integrated Astrophysical Modeling
}

\author{
T. A. Weaver, R. G. Eastman, P. Dubois, P. G. Eltgroth \\ N. Gentile, K. Jedamzik, J. R. Wilson
}

June 3, 1997

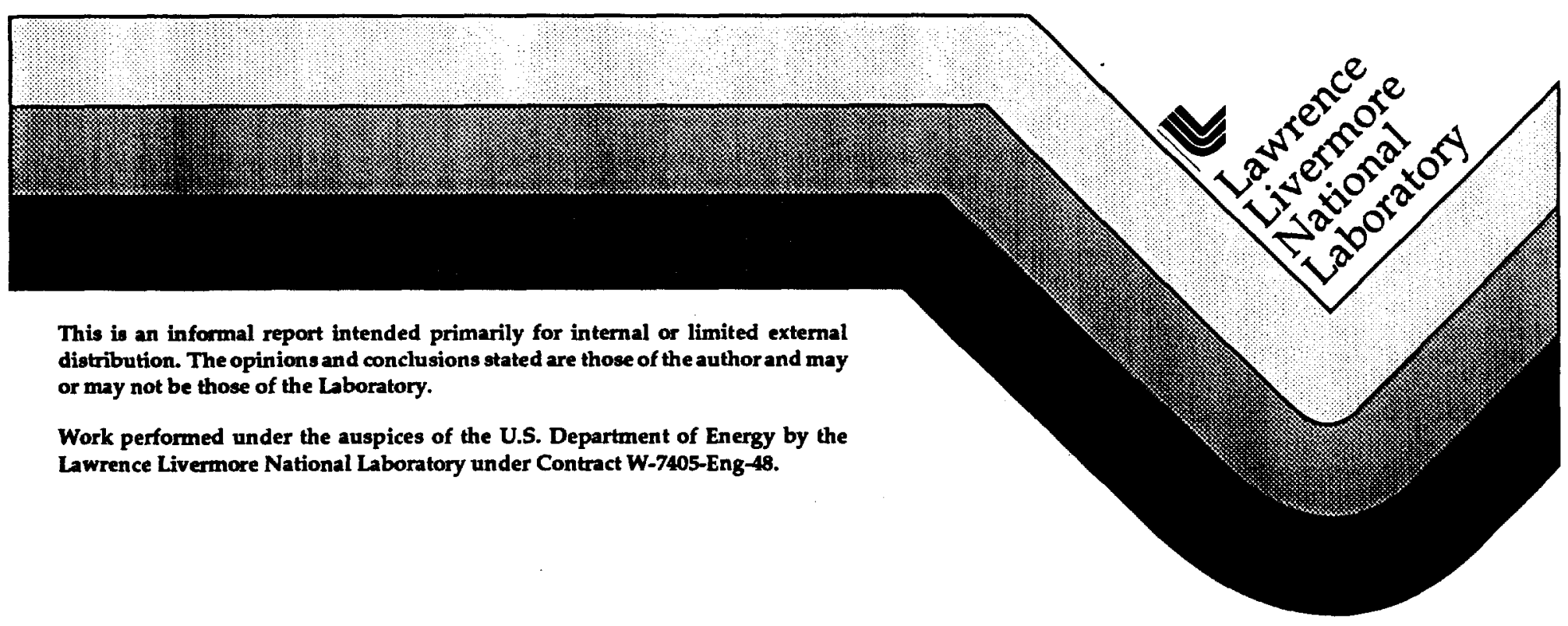




\section{DISCLAIMER}

This document was prepared as an account of work sponsored by an agency of the United States Government. Neither the United States Government nor the University of California nor any of their employees, makes any warranty, express or implied, or assumes any legal liability or responsibility for the accuracy, completeness, or usefulness of any information, apparatus, product, or process disclosed, or represents that its use would not infringe privately owned rights. Reference herein to any specific commercial product, process, or service by trade name, trademark, manufacturer, or otherwise, does not necessarily constitute or imply its endorsement, recommendation, or favoring by the United States Government or the University of California. The views and opinions of authors expressed herein do not necessarily state or reflect those of the United States Government or the University of California, and shall not be used for advertising or product endorsement purposes.

This report has been reproduced directly from the best available copy.

Available to DOE and DOE contractors from the Office of Scientific and Technical Information

P.O. Box 62, Oak Ridge, TN 37831

Prices available from (615) 576-8401, FTS 626-8401

Available to the public from the

National Technical Information Service

U.S. Department of Commerce

5285 Port Royal Rd.,

Springfield, VA 22161 


\title{
INTEGRATED ASTROPHYSICAL MODELING LDRD 94-ERP-044 FINAL REPORT
}

\author{
T.A. Weaver, R.G. Eastman, P. Dubois, P.G. Eltgroth, N. Gentile, K. Jedamzik, \\ and J:R. Wilson \\ Lawrence Livermore National Laboratory
}

In this project, we have developed prototype techniques for linking and extending a variety of astrophysical modeling capabilities, including those involving multidimensional hydrodynamics, complex transport, and flexibly-coupled equation-of state and nuclear reaction networks. As expected, this project is having both near-term payoffs in understanding complex astrophysical phenomena, as well as significant spin-offs in terms of people and ideas to related ASCI code efforts. Further development is continuing with programmatic funding. The core team includes several collaborators, both inside and outside the Laboratory.

Most of our work in the first part of this project was focused on the modularization, extension, and initial integration of 4 previously separate and incommensurate codes: the stellar evolution/explosion code KEPLER; the non-LTE spectral line transport code, EDDINGTON, used for modeling supernovae spectra; the 3-D smooth particle hydro code, PIP; and the discontinuous-finite-element, 3D hydro module from the ICF3D code. In particular, techniques for integration of 3D hydro with nuclear burn networks were developed, as were techniques for coupling and standardizing code modules -- with particular reference to operation in a parallel environment. In the process, we have invented and successfully implemented a new algorithm for treating transport in complex radiation fields with large discontinuities (PCAM -- Piecewise Continuous Analytic Method). We have made several extensions to the BASIS system to facilitate our work. We have also scoped an object- and network-oriented version of BASIS (tentatively called EQUIPE BASIS or TEAM BASIS) that promises robust and flexible operation over heterogeneous networks and other parallel computing environments.

We pursued the science applications of these modules as they were being 
integrated and extended, including calculations of the synthesis of the elements in massive stars of different initial metallicities and an analysis of the spectral evolution of Type II supernovae. Important new results that emerged from this phase of the work included: 1) a detailed understanding of the spectrum of Type II supernovae that allows them to be used with a increased degree of reliability to measure of the expansion rate and age of the universe; 2) the discovery that many isotopes above iron (previously thought to be made mostly in explosive $r-$ and $p$ processes) can be co-produced in essentially solar proportions with the s-process isotopes during vigorous hydrostatic nuclear burning: and 3) calculations of the synthesis of the elements in massive stars of different initial metallicities which (upon integration over plausible galactic histories) reproduce very well the currently observed pattern of solar abundances

After the transition of the code-integration part of this project into part of a broader ASCI effort during the project's second year, the remaining phase of the project was focused on code module development, testing, and application -- to both astrophysical and non-astrophysical problems. Some notable results of these efforts included:

1) The detailed comparison of the accuracy and efficiency of 3 distinct approaches to multidimensional hydrodynamics: a 2\&3-D piecewise-parabolic (PPM) Eulerian module, a 3-D discontinuous finite element hydro module, and a 3-D adaptive Lagrangian/Eulerian smooth-particle hydro module. In the test case of the highly non-linear flows involved in Richtmyer Meskov instabilities, the PPM approach was shown to be more accurate and efficient by a large factor relative to the other methods and to give good agreement with existing experimental data.

2) The application of our linked NLTE spectral-line transport and nuclear burn/implicit hydrodynamics capabilities to the study of supernovae. A definitive paper was completed on the use of Type II supernovae as cosmological distance indicators, indicating that they were likely to be the most accurate current method beyond the limited range where Cepheid variable stars can be used. For Type I supernovae, the same linked modeling techniques demonstrated that the shape of the light-curve can provide a diagnostic of the supernova's mass that is largely independent of opacity and explosion-energy differences.

3) The invention of an efficient, massively parallel method for solving linear systems of equations with direct application to ASCI and climate modeling codes. This algorithm involves a hierarchical reduction technique that provides an efficient, recursive way of solving 3-dimensional problems in terms of one-dimensional ones. 
4) The development of a 3-D multigroup radiation transport algorithm for use with 3-D Eulerian hydro methods such as PPM.

5) The continued development and application of a 3-D general-relativistic hydro module. This capability has been applied to calculating the behavior of close neutron star binaries, including their ability to generate gravitational waves potentially detectable with the LIGO detector now under construction. One surprising result of these calculations is that general relativistic interaction effects can cause otherwise stable neutron stars to collapse individually before merging.

6) Development of a 3-fluid, 1-dimensional hydrodynamical model for relativistic heavy ion collisions. This was applied to calculations of nuclear stopping in CERN experiments.

7) The pursuit of a variety of collaborative projects in cosmology, including: calculations of constraints on the properties of proto-galaxies, demonstration that it is unlikely that inhomogeneous nucleosynthetic processes can account for the variable $\mathrm{D} / \mathrm{H}$ ratios recently observed in clouds absorbing quaser radiation, and constraints on the effects of primordial magnetic fields on galaxy formation. 


\section{PUBLICATIONS}

"The Spectral Evolution of a Typical Type II Plateau Supernova," Ronald G.

Eastman, S. E. Woosley, and Thomas A. Weaver, BAAS 185, 3305 (1994)

"The Evolution and Explosion of Massive Stars. II. Explosive Hydrodynamics and Nucleosynthesis," S. E. Woosley and Thomas A. Weaver, Ap. J Supl. 101, 181 (1995).

"The Role of Mixing on the Appearance of Type Ib and Type Ic Supernova Spectra," Ronald G. Eastman and S. E. Woosley, BAAS 187, 9704 (1995).

“Type Ia and Type Ib Supernovae", S. E. Woosley, \& R. G. Eastman, in Proceedings of Menorca School of Astrophysics, eds. E. Bravo, R. Canal, J. Ibanez, and J. Isern, Societat Catalana de Fisica, 105, (1995).

"Gamma-Ray Producing Radioactivities from Supernova Explosions", R. D. Hoffman, S. E. Woosley, T. A. Weaver, F. X. Timmes, R. G. Eastman, and D. Hartmann, Proceedings Les Houches, 1994 meeting "The Gamma-Ray Sky with CGRO and Sigma", ed. M. Signore, P. Salati, \& G. Vedrenne, (Kluwer Acad. Pub.: Dordrect), (1995).

"The Influence of Nuclear Composition on the Electron Fraction in the Post-CoreBounce Supernova Environment," Gail C. McLaughlin, George M. Fuller, and James R. Wilson, Ap. J. 472, 440 (1996).

"The Atmospheres of Type II Supernovae and the Expanding Photosphere Method," Ronald G. Eastman, Brian. P. Schmidt, and Robert Kirshner, Ap. J. 466, 911 (1996).

"Instabilities in Close Neutron Star Binaries," James R. Wilson and Grant J. Mathews, Phys. Rev. Letters (1996).

"Relativistic Numerical Model for Close Neutron Star Binaries," James R. Wilson, Grant J. Mathews, and P. Marronetti, Phys. Rev. D 54, 1317 (1996). 
"A Hydrodynamical Model for Nuclear Stopping," James R. Wilson, Nuclear Physics A (1996).

"Binary Induced Neutron-Star Compression, Heating, and Collapse," Grant J. Mathews and James R. Wilson, Ap. J, in press (1997).

"Stochastic Isocurvature Baryon Fluctuations, Baryon Diffusion, and Primordial Nucleosynthesis," H. Kurki-Suonio, Karsten Jedamzik, and Grant J. Mathews, Ap.J 479, 31 (1997).

"Type II and Ib Supernova Progenitor Evolution and Hydrodynamics", S. E. Woosley, T. A. Weaver, \& R. G. Eastman, Proceedings of IAU Symp. 145 on Supernovae and Supernova Remnants, ed. R. McCray, (Cambridge Univ. Press) (1997).

"Spectrum Formation During the Plateau Phase of Type-IIp Supernovae" R. G. Eastman, Proceedings of IAU Symp. 145 on Supernovae and Supernova Remnants, ed. R. McCray, (Cambridge Univ. Press) (1997).

"Models of Type Ib Supernovae," S. E. Woosley \& R. G. Eastman, in Proceedings of the NATO ASI on Thermonuclear Supernovae, eds. P. RuizLapuente, R. Canal \& J. Isern (Kluwer), (1997).

"Radiation Transport in Type Ia Supernovae," R. G. Eastman, in Proceedings of the NATO ASI on Thermonuclear Supernovae, eds. P. RuizLapuente, R. Canal \& J. Isern (Kluwer), (1997).

"The Physics of Type Ia Supernova Lightcurves: I. Opacity and Diffusion," Phillip A. Pinto and Ronald G. Eastman, Ap. J. in press (1997). 


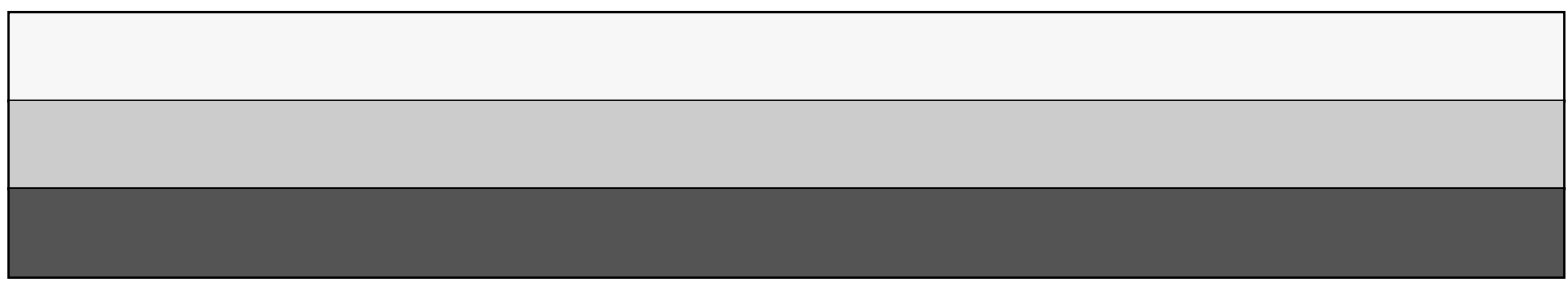

